\title{
Memory cross Volterra model for Doherty power amplifier with group delay mismatch
}

\author{
Caoyu Li ${ }^{1}$, Fei You ${ }^{1,}$ a), Jun Peng ${ }^{1}$, Ce Shen ${ }^{1}$, and Songbai He ${ }^{1}$
}

Abstract A Memory Cross Volterra model for Doherty power amplifiers (PA) with delay mismatch is presented in this letter. During the design process of Doherty power amplifier, gain, efficiency and operation bandwidth are mostly considered. Delay mismatch is difficult to avoid in this kind of dual-path circuit, which reduces the modeling performance of traditional behavioral models. The proposed Memory Cross Volterra Model (MCVM) is derived from the combination of three memory polynomial equations with delay mismatch. Simulation results show that the proposed MCVM has about $10 \mathrm{~dB}$ improvement in Normalized Mean Square Error (NMSE) compared to Generalized Memory Polynomial (GMP) with the same model complexity level as GMP. In a measurement experiment, a Doherty PA is tested at $3.45 \mathrm{GHz}$ with $20 \mathrm{MHz}$ LTE signal. Compared with GMP, the proposed MCVM has a maximum $2.5 \mathrm{~dB}$ improvement in Adjacent Chanel Power Ratio (ACPR), and the inverse modeling Normalized Mean Square Error (NMSE) is improved from -41.7 to -44.3 .

Keywords: behavioral model, power amplifier, Doherty, digital predistortion

Classification: Microwave and millimeter-wave devices, circuits, and modules

\section{Introduction}

Power Amplifiers (PA) are the critical power component in modern communication systems [1]. The efficiency of PA has a significant influence on the energy consumption of the whole system [2]. In recent decades, various advanced efficiency enhancement techniques are proposed in academics, such as harmonic control, Doherty amplifiers, outphasing and envelop tracking [3]. In these techniques, Doherty Power Amplifiers are preferred in commercial base-stations because of the good back-off efficiency which is suitable for communication signals with high Peak Average Power Ratio (PAPR).

In a Doherty PA, the input match network and output match network have to be designed carefully to make the two transistors work appropriately $[4,5]$. Especially the phase alignment which affects the power efficiency significantly [6, 7]. Many researchers have paid the phase adjustment much attention. However, the phase alignment is not equal to

${ }^{1}$ The authors are with School of Electronic Science and Engineering, University of Electronic Science and Technology of China, Chengdu 611731, China. Also with Yangtze Delta Region Institute of University of Electronic Science and Technology of China, Huzhou, 313000, China

a) feiyou@uestc.edu.cn

DOI: 10.1587/elex.18.20210064

Received February 2, 2021

Accepted February 4, 2021

Publicized February 22, 2021

Copyedited March 10, 2021 group delay alignment. The group delay mismatch may cause a complicated memory effect and bad linearity for Doherty PAs [8].

Linearization components such as digital predistortion (DPD) [1] are also an important subsystem of a transmitter. In recent years, novel applications bring new requirements and challenges for DPD system [9, 10, 11, 12]. Multiple kinds of behavioral models have been developed for modeling and predistortion in DPD system [1], such as Memory Polynomial [13, 14, 15], Dynamic Deviation Reduction (DDR) [16, 17, 18], Generalized Memory Polynomial (GMP) [19, 20, 21], piece-wise model [22, 23], and box oriented models [24]. These models have good performance for most PAs. These models do not focus on the nonlinearity with a group delay mismatch. Deep Neural Network-Based Models are reported to have good performance for Doherty amplifiers [25, 26] However, a large number of coefficients of Deep Neural Network Model (more than 1000) make these kinds of models difficult to be applied in real application due to the hardware and computing consumption.

This letter proposes a Memory Cross Volterra Model which is suitable for nonlinear systems with group delay mismatch. The model is derived from the combination of RF form memory polynomial with different group delays. Simulations and experiments are performed to prove the validity of the MCVM.

\section{Memory cross Volterra model}

Assume the two nonlinear branches have different group delays. The combination of these two signals also has a weak static nonlinear effect due to the non-ideal power combination. The nonlinear process of each branch is represented by RF form memory polynomial model. The output in RF expression of this system is given by:

$$
\begin{gathered}
\tilde{y}=\sum_{k_{0}=1}^{K_{0}}\left(a_{1} \widetilde{y_{1}}+a_{2} \widetilde{y_{2}}\right)^{k_{0}} \\
\widetilde{y_{1}}=\sum_{k_{1}=1}^{K_{1}} \sum_{m_{1}=0}^{M_{1}} b_{k_{1}, m_{1}} \widetilde{x}\left(n-m_{1}\right)^{k_{1}} \\
\widetilde{y_{2}}=\sum_{k_{2}=1}^{K_{2}} \sum_{m_{2}=M_{s}}^{M_{s}+M_{2}} b_{k_{2}, m_{2}} \tilde{x}\left(n-m_{2}\right)^{k_{2}}
\end{gathered}
$$

where $y_{1}$ and $y_{2}$ are the nonlinear output of branch 1 and branch 2. $M_{1}$ and $M_{2}$ are the memory depth of each branch. 
$M_{S}$ is the delay factor of the two branch. In order to derive the base-band expression of this system, let:

$$
\widetilde{x}=\operatorname{Re}\left\{e^{j w_{0} n} x(n)\right\}=\frac{e^{j w_{0} n} x(n)+e^{-j w_{0} n} x^{*}(n)}{2}
$$

Substitute Eq. (4) into Eq. (2) and Eq. (3). To simplify the expression, let $\xi_{m_{1}}=x\left(n-m_{1}\right)$ and $\psi_{m_{2}}=x\left(n-m_{2}\right)$

$$
\begin{array}{r}
y=\sum_{q_{2}=0}^{K_{2}} \sum_{q_{1}=0}^{K_{2}} \sum_{p_{2}=0}^{K_{1}} \sum_{p_{1}=0}^{K_{1}} \sum_{m_{2}=M_{s}}^{M_{s}+M_{2}} \sum_{m_{1}=0}^{M_{1}} b_{m_{1} m_{2} p_{1} p_{2} q_{1} q_{2}} \\
\xi_{m_{1}}^{p_{1}}\left(\xi_{m_{1}}^{*}\right)^{p_{2}} \psi_{m_{2}}^{q_{1}}\left(\psi_{m_{2}}\right)^{q_{2}} e^{j w n\left(p_{1}+q_{1}-p_{2}-q_{2}\right)}
\end{array}
$$

This is a kind of subseries of Volterra series [1]. The next step is to choose the proper terms, which have the major nonlinear influence in the Eq. (5). Criterion 1 is that the terms represent the component at the carrier frequency. Criterion 2 is that the major memory depth is related to the shorter delay. Select terms based on the criterion. After removing the carrier term $e^{j w n}$, the base-band expression of the proposed Memory Cross Volterra Model is given by:

$$
y=\sum_{d=1}^{4} \sum_{k=0}^{K} \sum_{m_{1}=0}^{M_{1}} \sum_{m_{2}=M_{s}}^{M_{s}+M_{2}} b_{m_{1} m_{2} k d} \xi_{m_{1}} B_{m_{1} m_{2} d}^{k}
$$

where the definition of $B_{m_{1} m_{2} d}$ is given by: $B_{m_{1} m_{2} 1}=$ $\xi_{m_{1}} \xi_{m_{1}}^{*}, B_{m_{1} m_{2} 2}=\xi_{m_{1}} \psi_{m_{2}}^{*}, B_{m_{1} m_{2} 3}=\xi^{*} \psi_{m_{2}}, B_{m_{1} m_{2} 4}=$ $\psi_{m_{2}} \psi_{m_{2}}^{*}$

\section{Simulation results}

In this section, simulations are performed to verify the nonlinear modeling ability of the proposed MCVM. The block diagram of the simulation is shown in Fig. 1. Saleh models [27] are applied to represent the nonlinear effect of a complex nonlinear system with delay mismatch. Two Saleh models Eq. (7) stand for the transistors of peak amplifier and carrier amplifier. Another Saleh model that has weak nonlinear stands for the non-ideal effect of the combiner. Extra loop delay is added in one of $y_{2}$ branch. In the simulation, the $x(n)$ is an LTE signal with $20 \mathrm{MHz}$ bandwidth. The sample speed of $x(n)$ is $122.88 \mathrm{Msa} / \mathrm{s}$. For comparison, Memory Polynomial (MP) [13] and Generalized Memory Polynomial (GMP) Models [19] are also applied to model the output of the nonlinear system. The model coefficients for all three models are calculated by the Least Square algorithm [1].

$$
y(n)=\frac{a_{1}|x(n)|}{1+b_{1}|x(n)|^{2}} \exp \left(\psi+\frac{a_{2}|x(n)|^{2}}{1+b_{2}|x(n)|^{2}}\right)
$$

The nonlinear order and memory depth settings of MP are 8 and 4 . The nonlinear order and memory depth of GMP are 8 and 4. Lagging and leading depth are both 2 . The parameter setting of MCVM is given as following: The

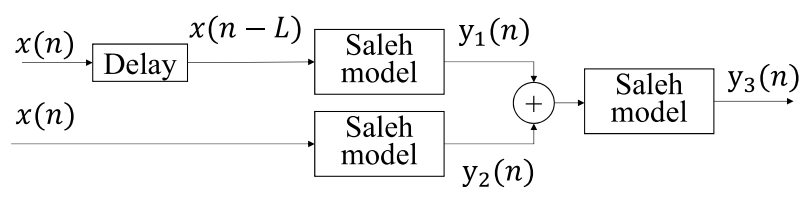

Fig. 1 Block diagram of simulation

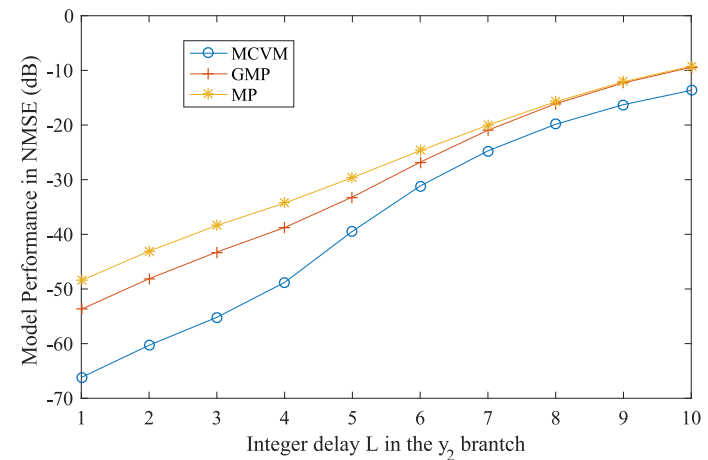

Fig. 2 Simulation result in NMSE with different integer delay of $y_{2}$ branch.

nonlinear order $K$ is 4 (the equivalent highest polynomial order is 8 because $B_{m_{1} m_{2} d}$ is a 2 order term). The memory depth of the first and second branches $\left(M_{1}\right.$ and $\left.M_{2}\right)$ are 3 and 1. The branch delay $\left(M_{S}\right)$ is changing with the simulation delay $L$. The coefficient number of MP, GMP, and MCVM are 81,160 and 160 correspondingly.

The forward modeling performance in Normalized Mean Square Error (NMSE) with different branch delay $L$ shown in Fig. 2. All three models show an accuracy reduction with the increment of the branch delay. The proposed MCVM has better NMSE than GMP and MP when $L$ is changed from 1 to 10 . However, when the $L$ is larger than 6 , all these three models shows not enough modeling ability for such a complicated nonlinear system.

\section{Experimental results}

Digital predistortion experiments are performed to prove the performance of the proposed MCVM. A symmetry Doherty PA with post-matching network is applied as the device under test (DUT) in this experiment. Two transistors are both CGHV27015 from Cree. The operating frequency bandwidth of the PA is $3.3 \mathrm{GHz}$ to $3.6 \mathrm{GHz}$. The tested PA performs a drain efficiency of over $40 \%$ in a back-off range of $7 \mathrm{~dB}$ and a gain about $12 \mathrm{~dB}$ in the operating frequency range. The peak output power is $43.5 \mathrm{dBm}$ when tested by continuous wave $(\mathrm{CW})$. R\&S SMA200A vector signal generator generates a $20 \mathrm{MHz}$ LTE signal with a sample speed $122.88 \mathrm{Msa} / \mathrm{s}$, which has a peak to average power ratio (PAPR) of $7 \mathrm{~dB}$. The average output power of PA is set to $32.0 \mathrm{dBm}$ and $35.7 \mathrm{dBm}$. Signal receiver is spectrum analyzer R\&S FSW 43 with $80 \mathrm{MHz}$ observation bandwidth of intermediate frequency (IF) filter.

Iterative learning control is a convenient tool to evaluate the linearization ability of a behavioral model [28, 29, 30]. The ILC signal can be considered as an ideal DPD signal of the tested PA. After training the ILC signal of the DUT, three models (MP, GMP and MCVM) are employed to fit the ILC signal by the LS algorithm. Due to the observation bandwidth limitation, a band-limited modeling method by filter is applied [31, 32]. Because there are two measurements with different output power, the linearization results are given separately. The DPD linearization performance with $32.0 \mathrm{dBm}$ output power is illustrated in Fig. 3. Details about the ACPR are given in Table I. With PA output power 


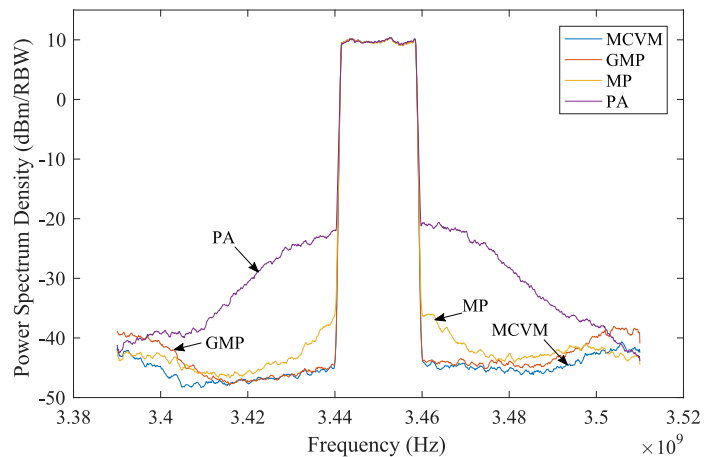

Fig. 3 Spectrum of experimental results with a output power of $32.0 \mathrm{dBm}$.

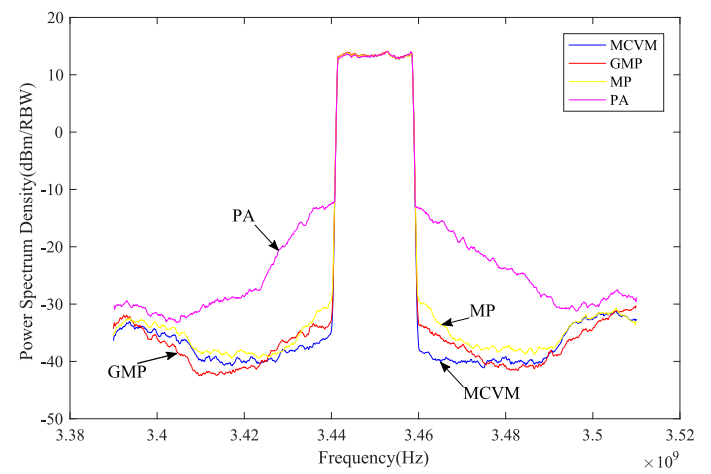

Fig. 4 Spectrum of experimental results with a output power of $35.7 \mathrm{dBm}$.

Table I Model performance comparison when the PA output power is $32.0 \mathrm{dBm}$

\begin{tabular}{c|cccc|cc}
\hline $\begin{array}{c}\text { ACPR } \\
(\mathrm{dB})\end{array}$ & $\begin{array}{c}\text { Alt } \\
\text { lower }\end{array}$ & $\begin{array}{c}\text { Adj } \\
\text { lower }\end{array}$ & $\begin{array}{c}\text { Adj } \\
\text { upper }\end{array}$ & $\begin{array}{c}\text { Alt } \\
\text { upper }\end{array}$ & $\begin{array}{c}\text { Coefficient } \\
\text { number }\end{array}$ & $\begin{array}{c}\text { NMSE } \\
(\mathrm{dB})\end{array}$ \\
\hline PA & -47.5 & -36.0 & -33.0 & -42.4 & - & - \\
\hline MP & -54.4 & -50.7 & -49.0 & -51.6 & 28 & -40.7 \\
\hline GMP & -54.1 & -54.8 & -53.2 & -52.2 & 175 & -41.7 \\
\hline Proposed & -56.5 & -55.2 & -53.8 & -53.9 & 168 & -44.3 \\
\hline
\end{tabular}

Table II Model performance comparison when the PA output power is $35.7 \mathrm{dBm}$

\begin{tabular}{c|cccc|cc}
\hline $\begin{array}{c}\text { ACPR } \\
(\mathrm{dB})\end{array}$ & $\begin{array}{c}\text { Alt } \\
\text { lower }\end{array}$ & $\begin{array}{c}\text { Adj } \\
\text { lower }\end{array}$ & $\begin{array}{c}\text { Adj } \\
\text { upper }\end{array}$ & $\begin{array}{c}\text { Alt } \\
\text { upper }\end{array}$ & $\begin{array}{c}\text { Coefficient } \\
\text { number }\end{array}$ & $\begin{array}{c}\text { NMSE } \\
(\mathrm{dB})\end{array}$ \\
\hline PA & -43.5 & -29.9 & -30.5 & -40.3 & - & - \\
\hline MP & -49.4 & -47.6 & -47.2 & -48.4 & 28 & -38.4 \\
\hline GMP & -52.7 & -49.2 & -50.0 & -51.0 & 175 & -42.0 \\
\hline Proposed & -50.6 & -51.0 & -52.5 & -49.1 & 168 & -42.8 \\
\hline
\end{tabular}

of $35.7 \mathrm{dBm}$, the linearization results are given by Fig. 4 and Table II. From Fig. 3, Fig. 4, Table I and Table II, the proposed MCVM has better ACPR than MP and GMP. Compared with GMP, the MCVM has 0.6-2.5 dB improvement with the same level of coefficient number. The NMSE between the ILC signal and the modeled ILC signal by the three model is also given in Table I and Table II, which further proof the modeling ability of the proposed MCVM.

\section{Conclusion}

In this letter, a memory cross Volterra model is proposed for behavior modeling and linearization of RF PA with loop delay mismatch. In the simulation, a complex nonlinear system is built with nonlinear delay mismatch. Forward mod- eling ability is evaluated by simulation. The performance of GMP and MP model are compared both in simulation and experimental results. The forward modeling ability and linearization performance of the proposed MCVM are better than GMP with the same level coefficients. For future issues, the performance of MCVM with other complicated systems $[33,34,35]$ with nonlinear loop combinations can be studied.

\section{Acknowledgments}

This work is supported by the National Key R\&D Program of China (2018YFB1802004), and partly by China Postdoctoral Science Foundation under Grant 2019M663472 and National Natural Science Foundation of China under Grant 62001082 .

\section{References}

[1] F.M. Ghannouchi, et al.: Behavioral Modeling and Predistortion of Wideband Wireless Transmitters (Wiley, Hoboken, NJ, USA, 2015) (DOI: 10.1002/9781119004424).

[2] L. Guan and A. Zhu: "Green communications: digital predistortion for wideband RF power amplifiers," IEEE Microw. Mag. 15 (2014) 84 (DOI: 10.1109/MMM.2014.2356037).

[3] S.C. Cripps: Power Amplifiers for Wireless Communications (Artech House Microwave Library, 2006) 2nd ed. (DOI: 10.1109/MMW. 2000.823830).

[4] W. Shi, et al.: "Broadband continuous-mode Doherty power amplifiers with noninfinity peaking impedance," IEEE Trans. Microw. Theory Techn. 66 (2018) 1034 (DOI: 10.1109/TMTT.2017.2749224).

[5] J. Xia, et al.: "Design of a 12dB back-off asymmetric Doherty power amplifier using reactive output impedance," IEICE Electron. Express 17 (2020) 20200156 (DOI: 10.1587/elex.17.20200156).

[6] Z. Cheng, et al.: "A Doherty power amplifier with extended efficiency and bandwidth," IEICE Electron. Express 14 (2017) 20170188 (DOI: 10.1587/elex.14.20170188).

[7] L. Zhan, et al:: "Linearization of power amplifiers with mismatched output impedance using on-line digital pre-distortion structure," IEICE Electron. Express 16 (2019) 20190207 (DOI: 10.1587/elex. 16.20190207).

[8] Y.Zhao, et al.: "Linearity improvement of HBT-based Doherty power amplifiers based on a simple analytical model," 2006 IEEE MTT-S International Microwave Symposium Digest (2006) 877 (DOI: 10.1109/ MWSYM.2006.249832).

[9] C.-W. Park, et al.: "A new digital predistortion technique for analog beamforming systems," IEICE Electron. Express 13 (2016) 20150998 (DOI: 10.1587/elex.13.20150998).

[10] Y. Bu, et al.: "A robust digital predistortion algorithm for power amplifiers," IEICE Electron. Express 11 (2014) 20130759 (DOI: 10.1587/elex.10.20130759).

[11] J. Jeong: "New digital predistortion technique of RF power amplifiers for wideband OFDM signals," IEICE Electron. Express 9 (2012) 326 (DOI: 10.1587/elex.9.326).

[12] L. Zhan, et al.: "Linearization of power amplifiers with mismatched output impedance using on-line digital pre-distortion structure," IEICE Electron. Express 16 (2019) 20190207 (DOI: 10.1587/elex. 16.20190207).

[13] J. Kim and K. Konstantinou: "Digital predistortion of wideband signals based on power amplifier model with memory," Electronics Letters 37 (2001) 1417 (DOI: 10.1049/el:20010940).

[14] B. Song, et al:: "Dynamic deviation memory polynomial model for digital predistortion," Electronics Letters 53 (2017) 606 (DOI: 10.1049/el.2017.0226)

[15] Z. He, et al.: "Digital predistortion of power amplifiers based on compound memory polynomial model," IEICE Electron. Express 10 (2013) 20130687 (DOI: 10.1587/elex.10.20130687).

[16] A. Zhu, et al.: "Open-loop digital predistorter for RF power am- 
plifiers using dynamic deviation reduction-based Volterra series," IEEE Trans. Microw. Theory Techn. 56 (2008) 1524 (DOI: 10.1109/ TMTT.2008.925211).

[17] A. Zhu and J.C. Pedro: "Distortion evaluation of RF power amplifiers using dynamic deviation reduction based Volterra series," 2007 IEEE/MTT-S International Microwave Symposium (2007) 965 (DOI: 10.1109/MWSYM.2007.380179).

[18] L. Guan and A. Zhu: "Simplified dynamic deviation reduction-based Volterra model for Doherty power amplifiers," 2011 Workshop on Integrated Nonlinear Microwave and Millimetre-Wave Circuits (2011) 1 (DOI: 10.1109/INMMIC.2011.5773325).

[19] D.R. Morgan, et al.: "A generalized memory polynomial model for digital predistortion of RF power amplifiers," IEEE Trans. Signal Process. 54 (2006) 3852 (DOI: 10.1109/TSP.2006.879264).

[20] Y.-J. Liu, et al.: "A robust augmented complexity-reduced generalized memory polynomial for wideband RF power amplifiers," IEEE Trans. Ind. Electron. 61 (2014) 2389 (DOI: 10.1109/TIE.2013.2270217).

[21] F. Mkadem, et al.: "Multi-band complexity-reduced generalizedmemory-polynomial power-amplifier digital predistortion," IEEE Trans. Microw. Theory Techn. 64 (2016) 1763 (DOI: 10.1109/TMTT. 2016.2561279).

[22] X. Kong, et al.: "A special complex-valued simplicial canonical piecewise linear function for amplifier and predistorter nonlinearity representation," IEICE Electron. Express 8 (2011) 1556 (DOI: 10.1587/elex.8.1556).

[23] L. Zhang and Y. Feng: "Three-dimensional power segmented tracking for adaptive digital pre-distortion," IEICE Electron. Express 13 (2016) 20160711 (DOI: 10.1587/elex.13.20160711).

[24] C. Chen et al.: "One-step model extraction method for joint polynomial/interpolating lookup-table two-box nonlinear model of power amplifier," IEICE Electron. Express 15 (2018) 20180634 (DOI: 10.1587/elex.15.20180634).

[25] R. Hongyo, et al.: "Deep neural network-based digital predistorter for Doherty power amplifiers," IEEE Microw. Wireless Compon. Lett. 29 (2019) 146 (DOI: 10.1109/LMWC.2018.2888955).

[26] Z. Liu, et al.: "Digital predistortion for wideband wireless power amplifiers using real-value neural network with attention mechanism," 2019 Photonics \& Electromagnetics Research Symposium Fall (PIERS - Fall) (2019) 3294 (DOI: 10.1109/PIERS-Fall48861. 2019.9021452).

[27] A.A.M. Saleh: "Frequency-independent and frequency-dependent nonlinear models of TWT amplifiers," IEEE Trans. Commun. 29 (1981) 1715 (DOI: 10.1109/TCOM.1981.1094911).

[28] J. Chani-Cahuana, et al.: "Iterative learning control for RF power amplifier linearization,” IEEE Trans. Microw. Theory Techn. 64 (2016) 2778 (DOI: 10.1109/TMTT.2016.2588483).

[29] M. Schoukens, et al.: "Obtaining the preinverse of a power amplifier using iterative learning control," IEEE Trans. Microw. Theory Techn. 65 (2017) 4266 (DOI: 10.1109/TMTT.2017.2694822).

[30] J. Chani-Cahuana, et al.: "Digital predistortion parameter identification for RF power amplifiers using real-valued output data," IEEE Trans. Circuits Syst. II, Exp. Briefs 64 (2017) 1227 (DOI: 10.1109/ TCSII.2017.2686004).

[31] Y. Li, et al.: "Sampling rate reduction for digital predistortion of broadband RF power amplifiers," IEEE Trans. Microw. Theory Techn. 68 (2020) 1054 (DOI: 10.1109/TMTT.2019.2944813).

[32] L. Ding, et al.: "Digital predistortion using direct learning with reduced bandwidth feedback," 2013 IEEE MTT-S International Microwave Symposium Digest (MTT) (2013) 1 (DOI: 10.1109/ MWSYM.2013.6697388).

[33] C. Li, et al:: "Group digital predistortion with step uniformization for hybrid beamforming transmitters," submitted to IEEE Microw. Wireless Compon. Lett.

[34] C. Li, et al.: "Analog predistorter averaged digital predistortion for power amplifiers in hybrid beam-forming multi-input multi-output transmitter," IEEE Access 8 (2020) 146145 (DOI: 10.1109/ACCESS. 2020.3013965).

[35] P. Hao, et al.: "Independently tunable linearizer based on characteristic self-compensation of amplitude and phase," IEEE Access 7 (2019) 131188 (DOI: 10.1109/ACCESS.2019.2940722). 